\title{
Setting ART initiation targets in response to changing guidelines: The importance of addressing both steady- state and backlog
}

\author{
C Martin, ${ }^{1}$ MB ChB, DipHIVMan, DTM\&H; N P Naidoo, ${ }^{1}$ MPH; W D F Venter, ${ }^{1,2}$ FCP, MMed, DTM\&H, DipHIVMan; A Jaffer, ${ }^{1}$ MPH; \\ P M Barker, ${ }^{3,4} \mathrm{MB}$ ChB, MRCP, FAAP \\ ${ }^{1}$ Wits Reproductive Health and HIV Institute, School of Clinical Medicine, University of the Witwatersrand, Johannesburg, South Africa \\ ${ }^{2}$ Department of Medicine, School of Clinical Medicine, University of the Witwatersrand, Johannesburg, South Africa \\ ${ }^{3}$ Institute for Healthcare Improvement, Cambridge, Massachusetts, USA \\ ${ }^{4}$ Gillings School of Global Public Health, University of North Carolina at Chapel Hill, North Carolina, USA
}

Corresponding author: C Martin (cmartin@wrhi.ac.za)

\begin{abstract}
Background. Target setting is useful in planning, assessing and improving antiretroviral treatment (ART) programmes. In the past 4 years, the ART initiation environment has been transformed due to the change in eligibility criteria (starting ART at a CD4 ${ }^{+}$count $<350$ cells/ $\mu$ lv. $<200$ cells/ $\mu \mathrm{l}$ ) and the roll-out of nurse-initiated management of ART.

Objective. To describe and illustrate the use of a target-setting model for estimating district-based targets in the era of an expanding ART programme and changing $\mathrm{CD}^{+}$count thresholds for ART initiation.

Method. Using previously described models and data for annual new HIV infections, we estimated both steady-state need for ART initiation and backlog in a North West Province district, accounting for the shift in eligibility. Comparison of actual v. targeted ART initiations was undertaken. The change in $\mathrm{CD}^{+}$count threshold adds a once-off group of newly eligible patients to the pool requiring ART - the backlog. The steady-state remains unchanged as it is determined by the annual rate of new HIV infections in previous years.

Results. The steady-state need for the district was 639 initiations/month, and the backlog was 15388 patients. After the shift in eligibility in September 2011, the steady-state target was exceeded over several months with some backlog addressed. Of the total backlog for this district, $72 \%$ remains to be cleared.

Conclusion. South Africa has two pools of patients who need ART: the steady-state of HIV-infected patients entering the programme each year, determined by historical infection rates; and the backlog created by the shift in eligibility. The healthcare system needs to build longterm capacity to meet the steady-state need for ART and additional capacity to address the backlog.
\end{abstract}

S Afr Med J 2014;104(6):428-430. DOI:10.7196/SAMJ.7507

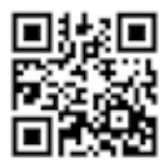

Target setting has been useful for both the National Department of Health $(\mathrm{NDoH})$ and supporting partners to plan, assess and improve the current performance of HIV testing and antiretroviral treatment (ART) programmes. Various models for estimating HIV testing and ART initiation need and coverage in South Africa (SA) have been popularised. ${ }^{[1-4]}$ However, less attention has been afforded to models which enable programme managers to effectively understand and plan for the ART need based on the local context, i.e. district and sub-district needs over the long term.

In the last 4 years, the ART initiation environment in SA has changed substantially due to policy amendments related to the roll-out of nurse-initiated management of ART ${ }^{[5]}$ and the change in the recommended $\mathrm{CD}^{+}$count threshold for initiation of ART, i.e. a shift from $<200$ cells $/ \mu$ lo $<350$ cells $/ \mu{ } .^{[6]}$ There remains a need for simple, practical tools which can be understood and applied at a local level to enable district planners to accurately plan and evaluate their HIV testing and ART programmes on an ongoing basis. Planning for both the sick and currently well sections of the HIV population in need of ART is imperative to ensure that the appropriate resources, including drugs, are allocated and utilised efficiently and effectively. Furthermore, planning should account for current performance relative to national and district-level targets. Leydon et al. ${ }^{[1]}$ proposed a model which uses local target setting in district healthcare facilities, accounting for the backlog of clients needing treatment.

We propose that the current ART need in SA can be planned for in terms of an annual 'steady-state' that adds new clients each year, and a single pool ('backlog') of currently asymptomatic patients who are eligible for treatment by virtue of the change in guidelines from a $\mathrm{CD} 4{ }^{+}$count threshold of $<200$ cells $/ \mu \mathrm{l}$ to $<350$ cells/ $\mu$ l. The healthcare system response is different for these two categories. Experience in supporting districts has shown that although some districts may meet their annual 'steady-state' ART initiation targets, districts have yet to meet the backlog need for ART created by the shift in $\mathrm{CD} 4^{+}$count thresholds.

\section{Objectives}

With the district health information system as a source, we used routine observational data from ART programmes in a North West Province (NWP) district ${ }^{[7]}$ to describe and illustrate the use of a target-setting model for estimating district-based targets in the era of an expanding ART programme and changing $\mathrm{CD}^{+}$count thresholds for ART initiation. 


\section{Methods}

Using two previously described models and tools developed by the Institute for Healthcare Improvement and the Wits Reproductive Health and HIV Institute, we estimated the steady-state and backlog need for ART initiation in a NWP district resulting from the shift in $\mathrm{CD}^{+}$count thresholds. ${ }^{[1,2]}$

These were calculated on the basis of a district population of $695933^{[8]}$ and antenatal HIV prevalence of $32.4 \%{ }^{[0]}$

Ethics approval was obtained from the Human Research Ethics Committee (Medical), University of the Witwatersrand (clearance no. M130206).

\section{Steady-state calculation}

The previously published highly active antiretroviral therapy (HAART) calculator is used to determine the steady-state need for ART initiations. ${ }^{[2]}$ This determines the number of incoming clients eligible for ART to be added to the programme each year, based on Actuarial Society of South Africa estimates for new HIV infections in previous years. It is estimated that it will take 5 years from the time of HIV acquisition to the time that an HIV-infected person's $\mathrm{CD}^{+}$cell count falls below 350 cells/ $\mu$, making him/ her eligible for ART. ${ }^{[10]}$ We used a simplified model to determine the steady-state for ART initiation prior to the change in $\mathrm{CD} 4^{+}$count thresholds, which assumed that patients with $\mathrm{CD} 4^{+}$counts of $<200$ cells $/ \mu \mathrm{l}$ are started on ART or die. Patients with $\mathrm{CD} 4^{+}$counts of 200 - 350 cells/ $\mu \mathrm{l}$ are started on ART, stay alive in the backlog 'pool' until they are started on treatment or die.

As described by Leydon et al. ${ }^{[1]}$ the steadystate need for ART initiation is determined by the historical annual infection rates and is not influenced by a change in $\mathrm{CD} 4^{+}$ count thresholds. The change in $\mathrm{CD}^{+}$ count thresholds does create a backlog, i.e. a one-time pool of additional patients with $\mathrm{CD}^{+}$counts $200-350$ cells $/ \mu \mathrm{l}$ who require ART. However, once this additional pool of patients has been initiated on ART, the annual requirements of the healthcare system for initiating new patients on treatment returns to a steady-state that is determined by new HIV infections in previous years. ${ }^{[1]}$

\section{Backlog calculation}

The change in ART initiation criteria results in a shortened interval from HIV acquisition to ART initiation: from 7 years if the threshold is 200 cells/ $\mu$ l to 5 years if the threshold is 350 cells $/ \mu \mathrm{l} .^{[10]}$ In the year that the $\mathrm{CD}^{+}$count threshold changes, an additional set of patients needing ART is added to the pool - in this case, an additional 2 years' worth of the steady-state need. This is the backlog. The model for calculating SA's steady-state and backlog is shown in Fig. 1. In this model, the system was at steady-state in 2010 when the $\mathrm{CD} 4^{+}$ count threshold was $200 \mathrm{cells} / \mu \mathrm{l}$. In that year in SA, 500 000 patients infected in $2003^{[11]}$ needed treatment. In 2011, the CD4 ${ }^{+}$ count threshold increased to 350 cells $/ \mu$, adding the backlog of patients from the 2004 and 2005 cohorts, which each added 500000 patients to the pool, as well as the new steady-state influx of 500000 patients from the 2006 cohort. In 2012, if the backlog was eliminated, the steady-state conditions returned with 500000 added from the 2007 cohort.

Using observational data at a district level, the proposed model was then applied to generate estimates of actual ART initiations in the district as compared with the steadystate and backlog targeted ART initiations as determined by the methods outlined above. In doing so, we propose that target setting at a district level incorporates two distinct steps: (i) the steady-state calculation; and (ii) the backlog estimate resulting in a comprehensive and more accurate district ART target.

\section{Results}

For this NWP district, the steady-state need was calculated at 639 initiations/month (7 669 per year). The backlog need created by the change in $\mathrm{CD}^{+}$count threshold was 15 388 patients. Since September 2011, after the shift in $\mathrm{CD}^{+}$count threshold, the steadystate target was exceeded over several months.
In those months, the backlog was decreased by the number of patients started on ART in excess of the steady-state target. The model assumes that ART initiations below the targeted steady-state threshold are initiations in patients with $\mathrm{CD} 4^{+}$counts $<200$ cells $/ \mu \mathrm{l}$ and that ART initiations above the steadystate target are initiations in patients with $\mathrm{CD}^{+}$counts between 200 cells $/ \mu \mathrm{l}$ and 350 cells $/ \mu$ l. In months where initiations fell below the steady-state target, the backlog was not affected. Using these assumptions, the backlog was decreased by 3335 initiations during months when the steady-state target was exceeded. The backlog was not affected when the initiation rate dropped below the steady-state target, since the great majority of patients treated under these conditions were assumed to have a $\mathrm{CD} 4^{+}$count of $<200$ cells $/ \mu$ l. Of the backlog, $72 \%$ remained, meaning that in this NWP district, $\sim 12000$ of the projected 15300 patients remained in the backlog pool awaiting initiation onto ART.

Fig. 2 depicts the relationship between actual ART monthly initiations, the steady-state monthly initiation target and backlog $\left(\mathrm{CD} 4^{+}\right.$ count 200 - 350 cells/ $\mu$ l) for the district. Actual ART initiations in the district were compared with targeted steady-state need. In the months where ART initiations exceeded the steadystate target, the backlog was reduced; however, during months where the steady-state target was not met, the backlog remained static.

\section{Discussion}

Practical tools and models that facilitate target setting at a district level enhance the response to the HIV epidemic. SA has two pools

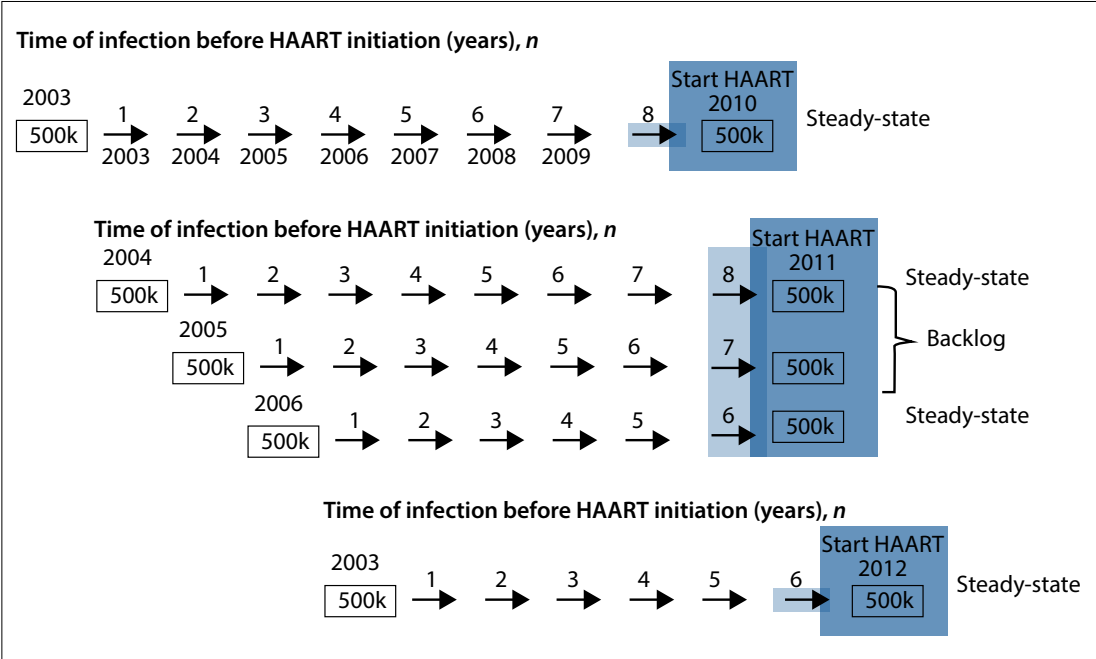

Fig. 1. A model for calculating South Africa's steady-state and backlog need for antiretroviral treatment (ART) initiation. Estimated number of new patients requiring ART initiation in 2010 (based on $\mathrm{CD}^{+}$count threshold of $200 \mathrm{cells} / \mu \mathrm{l}$ ), 2011 (during transition to new threshold of $350 \mathrm{cells} / \mu \mathrm{l}$ ) and 2012, assuming that the backlog created by the shift in eligibility is cleared. (HAART = highly active antiretroviral therapy; $500 k=500000$.) 


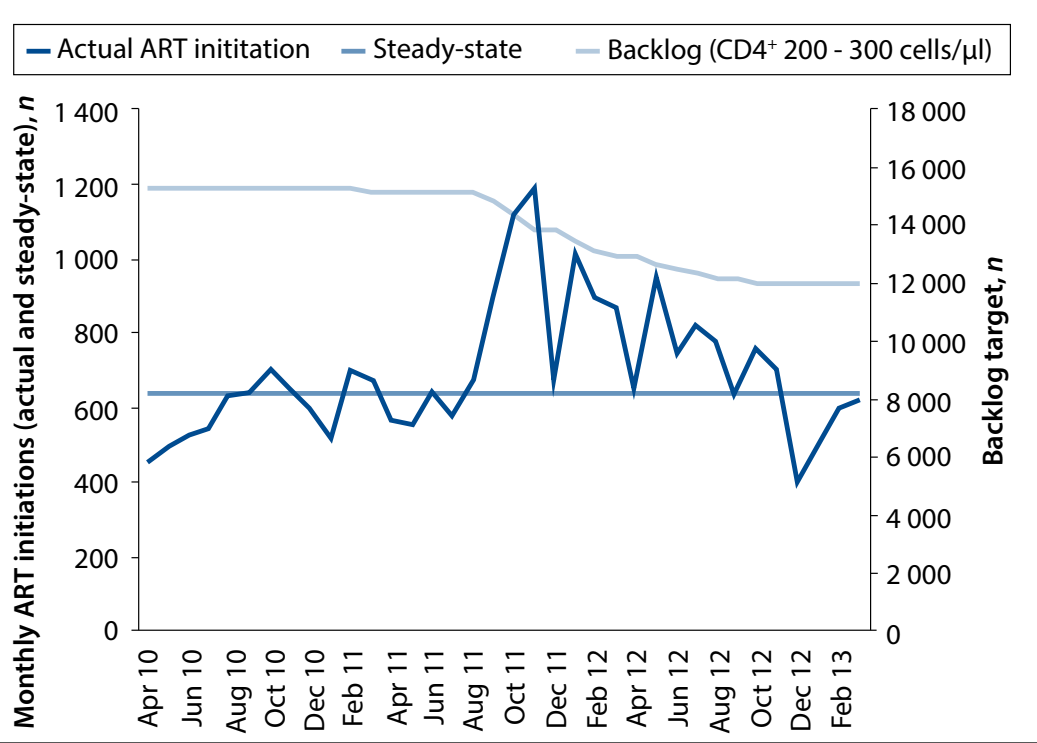

Fig. 2. The relationship between backlog and exceeding the steady-state target for the North West Province district. (ART = antiretroviral treatment.)

of HIV patients who need ART. Firstly, as described previously, there is a steady-state rate of HIV-infected patients who should be entering the ART programme each year. This is determined by the annual rate of new HIV infections in previous years. Secondly, a shift in the $\mathrm{CD}^{+}$count threshold causes a onetime additional backlog that is quantifiable and can be addressed by a temporary increase in system capacity. When steady-state rates are exceeded, the backlog can be worked down. Using this model, we estimated that in this NWP district the backlog decreased by $27 \%$ during the months when initiation rates exceeded the steady-state rate. Understanding the different approaches for steady-state and backlog initiations is needed to appropriately respond to the HIV care and treatment needs in each district. Each requires a different planning response.

Translated to SA as a whole, the healthcare system needs to build reliable longterm capacity to initiate and care for the $\sim 500000$ new patients per year generated by infections in past years. This annual number is expected to decrease in future years due to the declining annual infection rate (estimated 320000 in 2012) ${ }^{[11]}$ But the healthcare system also needs to be able to temporarily increase capacity to address the backlog of an additional one million patients in need of ART (Fig. 1) created by changes in the $\mathrm{CD}^{+}$count threshold. In addition, system planners need to build capacity to meet long-term chronic care needs of all of those started on ART each year.

The most recent data on ART initiation $(\sim 500000 \text { per year })^{[12]}$ suggest that SA is only providing sufficient ART initiation capacity to meet its steady-state needs. The projected additional one million patients who need treatment will undoubtedly place tremendous strain on the already overburdened healthcare system, which will need to add even greater capacity following any further increase in the $\mathrm{CD} 4{ }^{+}$count threshold.

\section{Study limitations}

The proposed model is based on assumptions that introduce some limitations to generalisability of the findings.

The model is based on estimates regarding population size, antenatal prevalence and ART initiation numbers. The number of new infections has decreased over the past 5 years from a high of 530000 in 2006 to 320000 in 2012. ${ }^{[1]}$ This means that the model, based on an average infection rate of 500000 per year, overestimates the steady-state need for future years. The model is simplistic in terms of its assumptions regarding the average time from HIV infection to ART need depending on $\mathrm{CD} 4^{+}$count thresholds, the consequences of not initiating $\mathrm{ART}$ at these thresholds, and the assumed $\mathrm{CD}^{+}$ counts of individuals who are being initiated on ART when the initiation targets are below or exceed the steady-state targets. The model has not included in the target the estimated $400000 \mathrm{HIV}$-infected pregnant women who need to receive antiretrovirals each year for the duration of pregnancy and breastfeeding, since these women are only transiently added to the pool of treated HIV-infected patients under the current $\mathrm{NDoH}$ policy. The policy to initiate all tuberculosis patients on ART will further add to the pool of patients in the backlog. Further, the model assumes that those patients who are lost to follow-up will either die or re-enter the treatment pool at a later date and are therefore not added back into the backlog estimate.

\section{Conclusion}

This paper highlights and describes a practical model for setting targets for ART initiation that can be applied at all levels of the healthcare system. It highlights the distinction between the steady-state need for ART initiation, and the backlog need created by changes in $\mathrm{CD} 4^{+}$count thresholds. Any further increase in the $\mathrm{CD} 4^{+}$count threshold with future World Health Organization recommendations would add further patients to the backlog, again with no effect on the steady-state requirements. Despite the limitations of the model, its simplicity allows it to have practical application and to provide a support tool to district planners in terms of the ART programme. Appropriate planning for the ART need, in a changing environment, is imperative to ensuring that targets are met, and that all eligible patients are identified, are able to access care and are initiated on ART.

\section{References}

1. Leydon NG, Venter F, Webster PD, Moleko W, Osih R, Barker PM. Achieving the HIV and AIDS National Strategic Plan: A practical calculator for local target setting in district health facilities. S Afr Med J 2010;100(7):420-424.

2. Barker PM, Venter F. Setting district-based annual targets for HAART and PMTCT - first step in planning effective for HAART and PMTCT - a first step in planning effective intervention for the HIV/AIDS epidemic. S Afr Med J

3. Adam MA, Johnson LF. Estimation of adult antiretroviral treatment coverage in South Africa. S Afr Med J 2009;99(9):661-667.

4. Johnson LF. Access to antiretroviral treatment in South Africa, 2004 - 2011. Southern African Journal of HIV Medicine 2012;13(1):22-27.

5. National Department of Health. Clinical Guidelines for the Management of HIV \& AIDS in Adults and Adolescents 2010. Pretoria: NDoH, 2010. http://www.sahivsoc.org/upload/ documents/Clinical_Guidelines_for_the_Management_of HIV_AIDS_in_Adults_Adolescents_2010.pdf (accessed 30 April 2013). 6. The Presidency, Republic of South Africa. Statement on the meeting of the South Afican National AIDS Corncil (SANAC). 12 August 2011. hitp./Nww.hepreside

7. Health Information Systems Programme. http://hisp.org/ (accessed 10 March 2014)

8. Statistics South Africa. South African National Population Census of 2011. https://www.statssa.gov.za/Census2011/ Products/Census_2011_Census_in_brief.pdf (accessed 10 March 2014).

9. National Department of Health. The 2010 National Antenatal Sentinel HIV \& Syphilis Prevalence Survey in South Africa. Pretoria: NDoH, 2011. http://www.gov.za/documents/ download.php?f=155559 (accessed 10 March 2014).

10. Pantaleo G, GraziosiC, Fauci AS. The immunopathogenesis of human immunodeficiency virus infection. N Engl J Med 1993;328(5):327335. [http://dx.doi.org/10.1056/NEJM199302043280508]

11. Actuarial Society of South Africa AIDS Committee. ASS A2008. Actuarial Society of South Africa AIDS Committee. ASSA2008. http://aids.actuarialsociety.org.za/ASSA2008-Model-3480.htm
(accessed 10 June 2013).

12. Pillay Y. Opening plenary. Southern African HIV Clinicians Society Conference. Cape Town, South Africa, $25-28$ November 2012.

Accepted 24 September 2013. 